\title{
EL CONSUMIDOR TURÍSTICO Y LA TEORÍA MICROECONÓMICA
}

\author{
TOURISM CONSUMER AND MICROECONOMIC THEORY
}

José Manuel Enrique Marsano Delgado

Universidad de San Martín de Porres, Perú

\section{RESUMEN}

Se analiza y evalúa el comportamiento microeconómico de la actividad turística desde el punto de vista del consumidor. Por ello, se aborda la definición del consumidor turístico y los factores que lo determinan.; además, se analiza el presupuesto del consumidor a través de la línea o recta de la restricción presupuestaria, y el campo de las curvas de indiferencia del consumidor turístico, explicando el punto de equilibrio del mismo, para finalmente inferir en el campo de la línea de restricción presupuestaria desde el punto de vista de los cambios y efectos en los ingresos, así como, en los precios de los servicios turísticos.

Palabras clave: Consumidor turístico, presupuesto, recta presupuestaria, curva de indiferencia, equilibrio del consumidor.

\begin{abstract}
This paper analyzes and evaluates the microeconomic behavior of tourism, from the consumer's point of view. In this sense, it tackles the definition of tourism consumer, the factors that determine it, analyzes the consumer budget through the line of budget constraint, addresses the field of tourism consumer indifference curves explaining its point of equilibrium, to finally infer in the field of the budget restriction line from the point of view of the changes and effects in the revenues and the prices of the tourism services.
\end{abstract}

Keywords: Tourism consumer, budget, budget line, indifference curve, consumer equilibrium.

Este es un artículo Open Access bajo la licencia Creative Commons Atribución-NoComercial-Compartirlgual 4.0

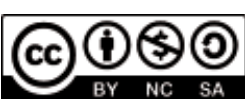




\section{Introducción}

Definiremos desde el punto de vista de la teoría económica al consumidor turístico como aquel individuo que, luego de haber satisfecho sus necesidades básicas, genera recursos excedentes que le permiten utilizarlos para ausentarse por un tiempo determinado del hábitat en el que se desenvuelve, a efectos de conocer otros lugares, ya sea de su propio país o del extranjero; gastando todo o parte de dichos excedentes en actividades turísticas de recreo, histórico culturales, de aventura, visita a familiares. Inclusive la Organización Mundial del Turismo (OMT), incluye como turista al hombre de negocio que viaja a un país por estos menesteres, siempre y cuando no exceda su permanencia a un tiempo determinado.

\section{Factores que determinan el consumo turístico}

Vemos que los factores que determinan el consumo turístico están sujetos a variables exógenas. Variables sobre las cuales no se puede ejercer ningún control en el consumo turístico. Por lo general, estos factores no están en forma directa relacionados con el turismo, pero influyen a no dudarlo, en la demanda de la actividad turística. Dentro de este grupo de factores podemos señalar los siguientes: la situación económica del país, la situación política, accidentes geofísicos y la estacionalidad, entre muchos otros. Sin embargo, nos encontramos con factores que dependen de variables endógenas, variables que en mayor o menor medida son controlables por los agentes económicos, como por ejemplo, la calidad de la oferta del destino turístico, la viabilidad de los accesos a los restos arqueológicos, la adecuada oferta de hospedaje y restauración, así como otros más.

\section{El presupuesto y la línea de restricción presupuestaria}

Marsano (2003) explica:

En la economía real, la mayoría de los individuos, cuentan con un ingreso fijo proveniente casi siempre de la remuneración de su trabajo en las unidades de producción, el mismo que lo pueden recibir en forma mensual, quincenal, o semanal. El caso es que el mismo constituye la fuente de ingresos, con los que las personas adquieren bienes y servicios elaborados por las distintas unidades de producción o empresas. A este concepto se le llama presupuesto de las personas.

En el presente documento definiremos la Línea de balance, Recta presupuestaria, o Restricción presupuestaria, como aquella dentro de lo que un individuo al que se le llama consumidor, en base a sus ingresos puede combinar una serie de alternativas de adquisición de bienes y servicios o canasta de bienes y servicios, disponiendo del total de sus ingresos entre la que incluimos la actividad turística.

A efectos de poder clarificar adecuadamente el concepto de la Recta Presupuestaria del Consumidor, partiremos del siguiente ejemplo: se asume que un individuo tiene una renta mensual de S/ 1.000, los mismos que tendrá que gastarlos en una determinada cantidad del bienes o servicios que definimos (A), u otra cantidad del bienes o servicios que definimos (B) y/o en su defecto, combinará la adquisición de una determinada cantidad del bienes y servicios (A) y otra de bienes y servicios (B). (Marsano, 2003) 
Tabla 1

Posibilidades de Consumo

\begin{tabular}{|c|c|c|c|c|c|}
\hline $\begin{array}{l}\text { Número de } \\
\text { alternativa }\end{array}$ & $\begin{array}{c}\text { Bienes o servicios A } \\
\text { cantidad posible de } \\
\text { consumo }\end{array}$ & $\begin{array}{l}\text { Precio de los } \\
\text { bienes o } \\
\text { servicios A }\end{array}$ & $\begin{array}{c}\text { Bienes o servicios B } \\
\text { cantidad posible de } \\
\text { consumo }\end{array}$ & $\begin{array}{c}\text { Precio de los } \\
\text { bienes o servicios } \\
\text { B }\end{array}$ & $\begin{array}{l}\text { Ingreso del } \\
\text { Consumidor } \\
\text { en soles }\end{array}$ \\
\hline 1 & 0 & 20 & 25 & 40 & 1.000 \\
\hline 2 & 10 & 20 & 20 & 40 & 1.000 \\
\hline 3 & 8 & 20 & 21 & 40 & 1.000 \\
\hline 4 & 6 & 20 & 22 & 40 & 1.000 \\
\hline 5 & 2 & 20 & 24 & 40 & 1.000 \\
\hline 6 & 50 & 20 & 0 & 40 & 1.000 \\
\hline
\end{tabular}

Fuente y elaboración propia

Del análisis de la Tabla 1 se puede concluir que, de las seis alternativas, las cuales a su vez contienen igual número de combinaciones, el total de adquisiciones de bienes o servicios, es igual al ingreso total del consumidor. Las posibilidades de adquisición de bienes quedan representadas por la siguiente ecuación:

$20 \times \mathrm{QA}+40 \times \mathrm{QB}=\mathrm{S} / .1000$ = RENTA PRESUPUESTARIA DEL CONSUMIDOR

Se podrá notar que la representación gráfica de dicha ecuación, la misma que representa la restricción presupuestaria del consumidor es lineal y la pendiente de la misma está representada por el valor absoluto de: diferencial QA / diferencial QB = 2 o por PB / PA = 2 (Ver Figura 1).

En donde:

QA: representa la cantidad adquirida del bien o servicios A

QB: representa la cantidad adquirida del bien o servicios B

PA: representa el precio del bien o servicios A

PB: representa el precio del bien o servicios B 
Figura 1. Recta presupuestaria.

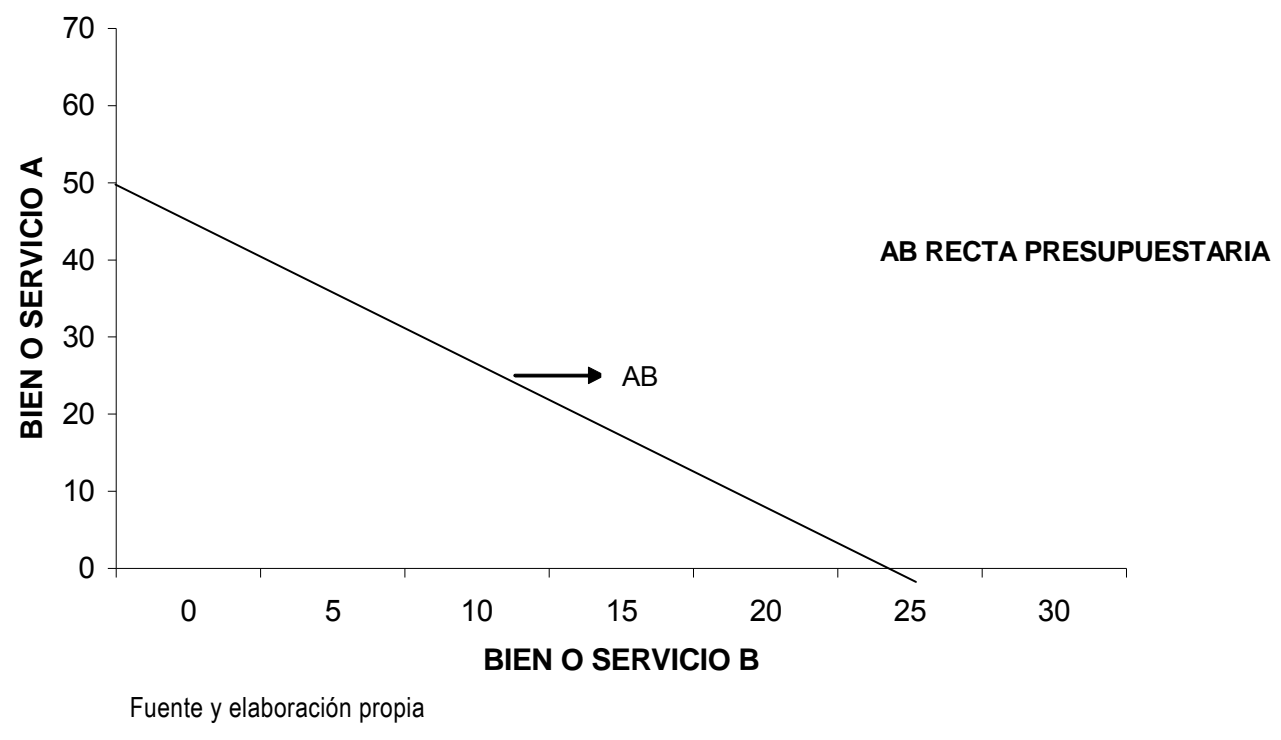

\section{La curva de indiferencia}

Marsano (2003) define desde el punto de vista económico, la curva de indiferencia de la siguiente manera:

Aquella curva que combina dos o más bienes y/o servicios, o canasta de bienes en mayor o menor cantidad de uno de ellos a determinados precios. Estas combinaciones representan bienes y/o servicios, en las que el consumidor se muestra indiferente a la cantidad de bienes o servicios; todas estas combinaciones le son igualmente deseables. Desde el punto de vista turístico, podemos asumir que cualquiera de los destinos turísticos le produce el mismo nivel de satisfacción.

Tabla 2

Combinaciones indiferentes para el consumidor

\begin{tabular}{ccc}
\hline Alternativa & $\begin{array}{c}\text { Bienes o servicios A } \\
\text { Cantidad posible de } \\
\text { consumo }\end{array}$ & $\begin{array}{c}\text { Bienes o servicios B } \\
\text { Cantidad posible de } \\
\text { consumo }\end{array}$ \\
\hline A & 60 & 10 \\
B & 30 & 20 \\
C & 20 & 30 \\
D & 15 & 40 \\
\hline
\end{tabular}

Fuente y elaboración propia 
Figura 2. Curva de Indiferencia.

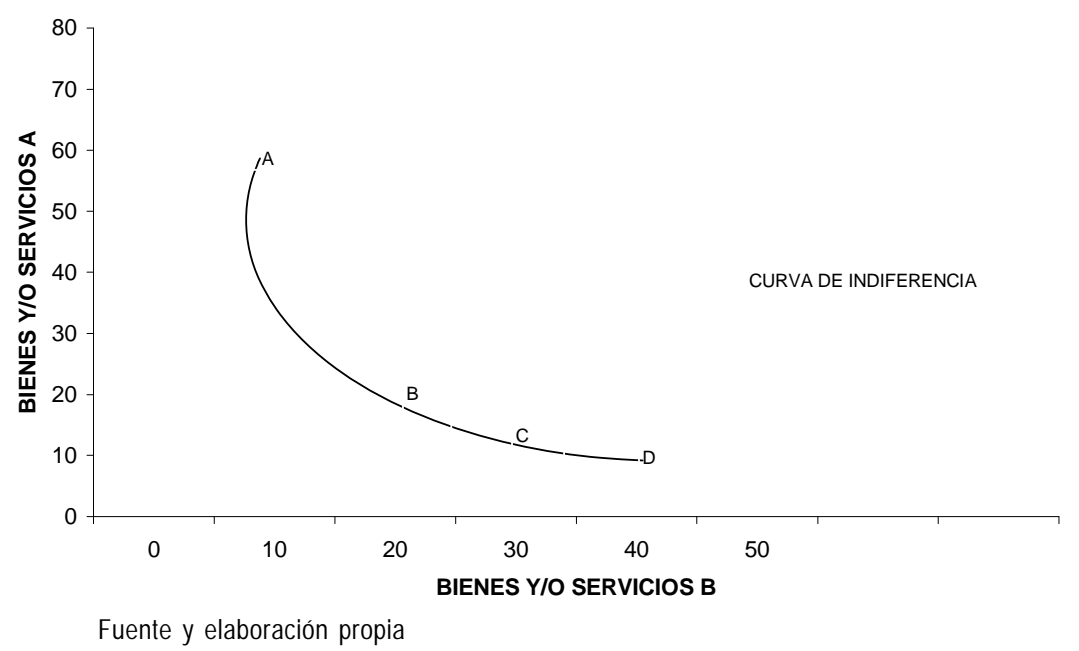

Como se puede apreciar, la adquisición de una mayor cantidad de un bien o servicio es compensado o renunciando a la adquisición de parte de la cantidad del otro bien o servicio. Al consumidor le da lo mismo, las alternativas A, B, C, o D; las mismas que se pueden observar en la Tabla 2 o en la Figura 2; el cual como se puede ver, es una curva de indiferencia; la misma que es convexa desde abajo, debido a la ley de sustitución, ya que al aumentar la cantidad que se posee de un bien, disminuye su relación de sustitución o la pendiente de la curva de indiferencia. Por otro lado, las combinaciones de ambos bienes o servicios le reportan la misma satisfacción al consumidor.

Marsano (2003), indica que las curvas de indiferencia son convexas, lo que permite inferir que a medida que un consumidor se vaya desplazando por ellas hacia la derecha, movimiento que en el caso de la Figura 2 implica un aumento en la cantidad adquirida del bien B y una disminución en las unidades del bien A. La tendencia de la curva es hacía la horizontalidad. Lo cual resulta ser cierto en el sector real, denominándose la Ley de Sustitución. Nordhaus y Samuelson (1996) señalan que «cuanto más escaso es un bien, mayor es su valor relativo de sustitución; su utilidad marginal aumenta en relación con el bien que se ha vuelto abundante». Esto se puede ver en la Tabla 2, o en la Figura 2. Así para desplazarse de A a B en el cuadro o la figura antes citados, el consumidor estará dispuesto a renunciar a 30 unidades del bien o servicio A para efectos de adquirir 10 unidades adicionales del bien o servicio B. No obstante ello, para desplazarse a C, requerirá sacrificar menos unidades del bien o servicio A, en este caso 10 para conseguir 10 unidades del bien $\mathrm{B}$. Y si se desplaza el consumidor a la alternativa D, se sacrificaran 5 unidades adicionales del bien o servicio A, para conseguir 10 unidades adicionales del bien B.

Si se unen los puntos de las alternativas A y B se observa que se forma una recta cuya pendiente en términos de valor absoluto es 3 , uniendo los puntos o coordenadas de las alternativas B y C la pendiente es 1 ; y si se unen las coordenadas de las alternativas C y D, la pendiente de la recta es 0,5. 
Estas pendientes vienen a ser en realidad las relaciones marginales de sustitución entre los bienes. Ahora bien, cuanto menor sean los movimientos en términos de magnitud entre un punto y otro de una curva de indiferencia, más se acercará la relación de sustitución a la pendiente real de la curva de indiferencia. Como corolario de lo antes expuesto Nordhaus y Samuelson (1996) señalan que se debe inferir lo siguiente:

La pendiente de una curva de indiferencia es la medida de las utilidades marginales relativas de los bienes o servicios, o de la relación de sustitución a la que cuando se trata de cambios muy pequeños, el consumidor estará dispuesto a cambiar un poco de un bien por un poco de otro.

Una curva de indiferencia, como la expuesta en la Figura 2, responde pues a la ley de sustitución. En la medida que aumenta la cantidad consumida de los bienes o servicios B, y disminuye la de los bienes o servicios A. Los bienes B tienden a abaratarse cada vez más a efectos de que el consumidor acepte una mayor cantidad de los bienes $\mathrm{B}$.

\section{El equilibrio del consumidor}

El consumidor encuentra el equilibrio en aquel punto de la Recta AB de Balance o Presupuestaria en la que es tangente con la curva de indiferencia más alta (Ver Figura 3). Si se analiza la figura, la curva de indiferencia es tangente a la recta de restricción presupuestaria en el punto o coordenadas $(20,10)$. En términos de geometría analítica se encuentra en equilibrio, lo cual como se sabe, viene a ser la relación entre el precio de los bienes o servicios B y la de los bienes o servicios A, y que es igual a la pendiente de la curva de indiferencia la que es igual al cociente de las utilidades marginales de los bienes A / bienes B. Es decir, la relación de sustitución, queda representada numéricamente por la siguiente relación: Precio de bienes B / Precio de bienes $\mathrm{A}=\mathrm{UM}$ bienes $\mathrm{A} / \mathrm{UM}$ bienes $\mathrm{B}$.

Figura 3. Equilibrio del consumidor.

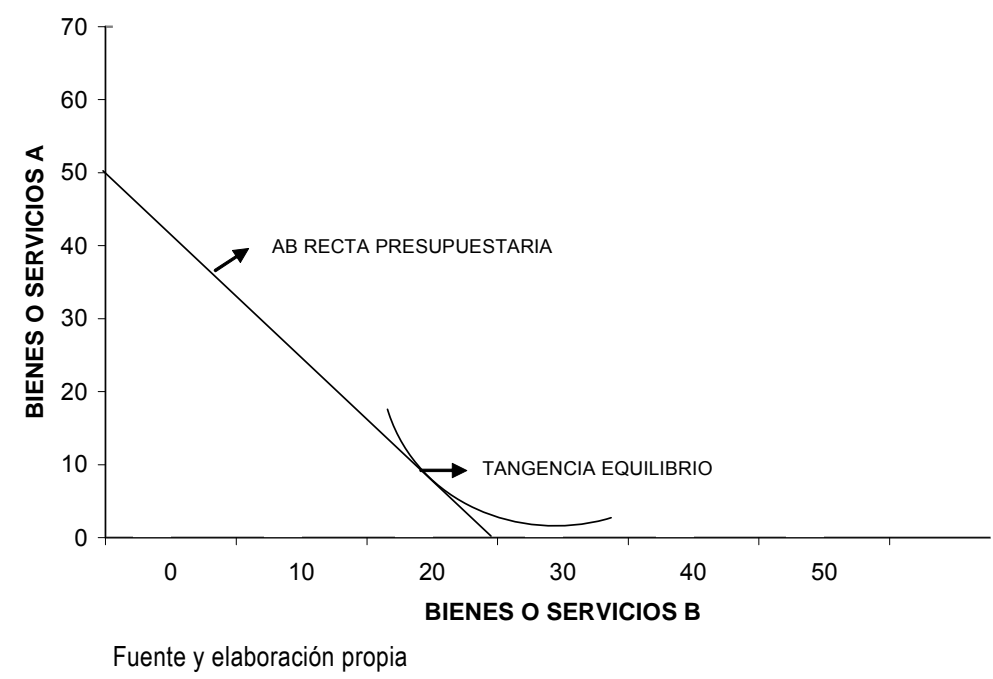




\section{La línea de restricción presupuestaria y los cambios en los ingresos y en los precios}

\section{Cambio en los ingresos}

Para explicar el efecto en el cambio de los ingresos, se parte de la premisa de que el consumidor, ve reducido sus ingresos en por ejemplo 40,0\%. Es decir, si antes sus ingresos mensuales eran de S/ 1.000, ahora estos son de S/ 600. Como se podrá notar, con la nueva capacidad de recursos la Recta de Restricción Presupuestaria, le permitirá adquirir una combinación menor de bienes o servicios de uno de ellos, teniendo en consideración de que el precio de los mismos ha permanecido constante.

Tabla 3

Cambio en los ingresos

\begin{tabular}{cccccc}
\hline Alternativa & $\begin{array}{c}\text { Bienes y servicios A } \\
\text { Cantidad posible de } \\
\text { consumo }\end{array}$ & $\begin{array}{c}\text { Precio de los } \\
\text { bienes y } \\
\text { servicios A }\end{array}$ & $\begin{array}{c}\text { Bienes o servicios B } \\
\text { Cantidad posible de } \\
\text { consumo }\end{array}$ & $\begin{array}{c}\text { Precio de los } \\
\text { bienes y } \\
\text { servicios B }\end{array}$ & $\begin{array}{c}\text { Ingresos del } \\
\text { Consumidor }\end{array}$ \\
\hline 1 & 0 & 20 & 15 & 40 & 600 \\
2 & 6 & 20 & 12 & 40 & 600 \\
3 & 4.8 & 20 & 12.6 & 40 & 600 \\
4 & 3.6 & 20 & 13.2 & 40 & 600 \\
5 & 1.2 & 20 & 14.4 & 40 & 600 \\
\hline
\end{tabular}

Fuente y elaboración propia

Figura 4. Cambio en los ingresos.

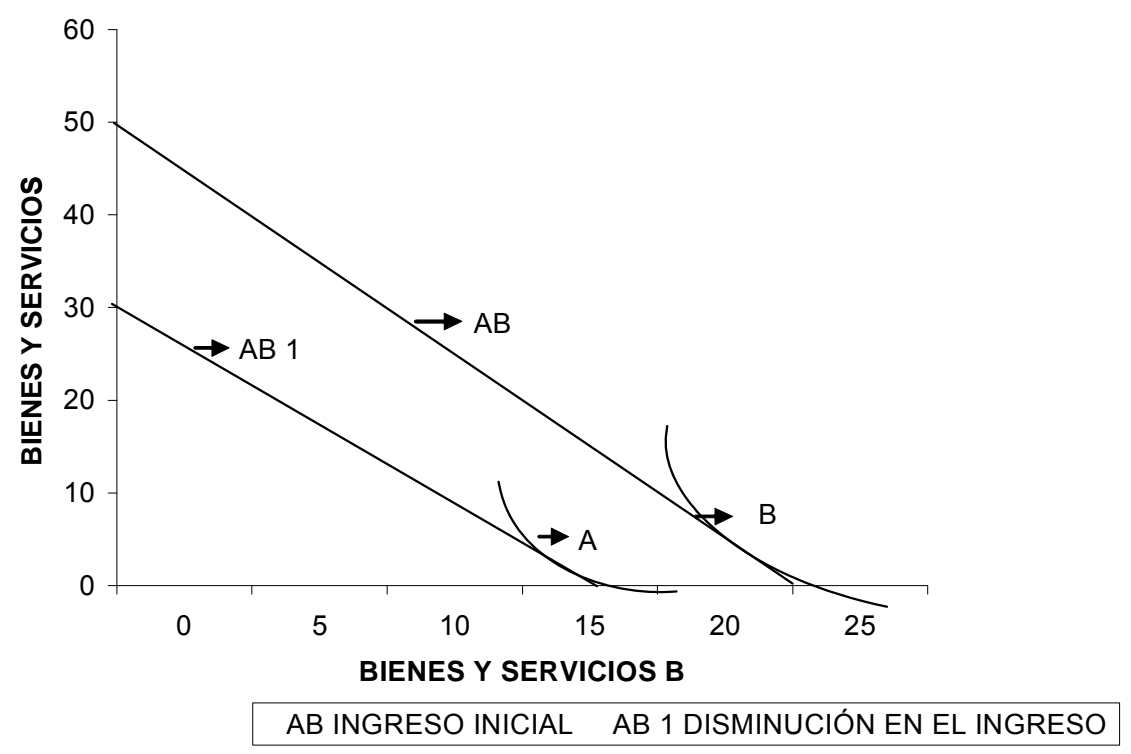

Fuente y elaboración propia 
Lo antes expuesto, se pude analizar en términos de geometría analítica (Ver Tabla 3 y Figura 4). Se debe recordar que cuando el consumidor recibía una suma de ingresos equivalentes a $S$ / 1.000, su recta de Balance $\mathrm{AB}$, le permitía adquirir, por ejemplo, exclusivamente 50 unidades de bienes A o 25 unidades de bienes B y encontraba su equilibrio en la curva de indiferencia B, la misma que era tangente a su Recta de Balance, en las coordenadas $(20,10)$. Es decir que en el punto de equilibrio, el consumidor está en capacidad de adquirir 20 unidades de bienes o servicios B a un precio unitario de S/ 40 y 10 unidades de bienes o servicios A al precio unitario de $\mathrm{S} / 20$. Si se efectuaban las correspondientes operaciones veíamos que el consumidor encontraba su equilibrio gastando S/ 800 en bienes y servicios B y S/ 200 en bienes y servicios A. La sumatoria de ambas adquisiciones lo situaba en su gasto máximo presupuestario de S/ 1.000.

Al haberse reducido los ingresos del consumidor a S/ 600, vemos que automáticamente la Recta de Balance se desplaza hacia el origen, siendo su nueva recta presupuestaria la línea $\mathrm{AB}$ 1, la misma que le permitirá al consumidor, por ejemplo, adquirir un máximo de bienes o servicios A, equivalente a 30 unidades, o un máximo de bienes B de 15 unidades. El consumidor encontrará una nueva curva de indiferencia A, que evidentemente será inferior a los niveles de satisfacción obtenidos en la curva de indiferencia B. Por otro lado, el consumidor encontrará su punto de equilibrio en las coordenadas $(12,6)$, en donde la nueva curva de indiferencia, es tangente con la Recta de Restricción Presupuestaria. Lo que equivale a señalar que en el nuevo punto de equilibrio, producto de una nueva Recta de Restricción Presupuestaria y de una nueva curva de indiferencia, estará en capacidad de adquirir 12 unidades de bienes o servicios B a un precio unitario de S/ 40 y 6 unidades de bienes o servicios A al precio unitario de S/ 20. Ello implica, que el consumidor gastará S/ 480 en bienes o servicios B y S/ 120 en bienes o servicios A. La sumatoria de ambas operaciones nos señala que el gasto total de la nueva combinación, en la nueva curva de indiferencia, será igual a S/ 600.

\section{Cambio en los precios}

Es indudable que el incremento en el precio de uno o de un conjunto de bienes o servicios ya sean del tipo A o B, redundará en una pérdida del poder adquisitivo, asumiendo que el ingreso del consumidor permanece constante. Ello se puede ver en la Tabla 4 y Figura 5, en donde, se observa que el precio de los bienes o servicios B se incrementó en un 100,0\%; mientras que el precio de los bienes o servicios A se mantuvo constante al igual que el ingreso del consumidor. En esta nueva situación, el consumidor podrá seguir consumiendo la misma cantidad de bienes o servicios A, mientras que verá reducida su capacidad de consumo de bien o servicios B. Es importante considerar ello, ya que si el consumidor decidiese gastar todo su dinero en bienes o servicios A, podrá seguir adquiriendo la cantidad de 50 unidades. Mientras que si decide gastar toda su renta en bienes o servicios B, solo podrá adquirir 12,5 unidades de bienes o servicios B. Esto significa una merma de un 50,0\% en la adquisición de dichos bienes. 
Tabla 4

Cambio en los precios

\begin{tabular}{ccccc}
\hline $\begin{array}{c}\text { Bienes y servicios A } \\
\text { Cantidades posibles } \\
\text { de consumo }\end{array}$ & $\begin{array}{c}\text { Precio de los } \\
\text { bienes y servicios } \\
\text { A }\end{array}$ & $\begin{array}{c}\text { Bienes o servicios B } \\
\text { Cantidades posibles } \\
\text { de consumo }\end{array}$ & $\begin{array}{c}\text { Precio de los } \\
\text { bienes y servicios } \\
\text { B }\end{array}$ & $\begin{array}{c}\text { Ingresos } \\
\text { del } \\
\text { Consumidor }\end{array}$ \\
\hline 0 & 20 & 12.5 & 80 & 1.000 \\
10 & 20 & 10 & 80 & 1.000 \\
8 & 20 & 10.5 & 80 & 1.000 \\
6 & 20 & 11 & 80 & 1.000 \\
2 & 20 & 12 & 80 & 1.000 \\
50 & 20 & 0 & 80 & 1.000 \\
\hline
\end{tabular}

Fuente y elaboración propia

Figura 5. Cambio en los precios.

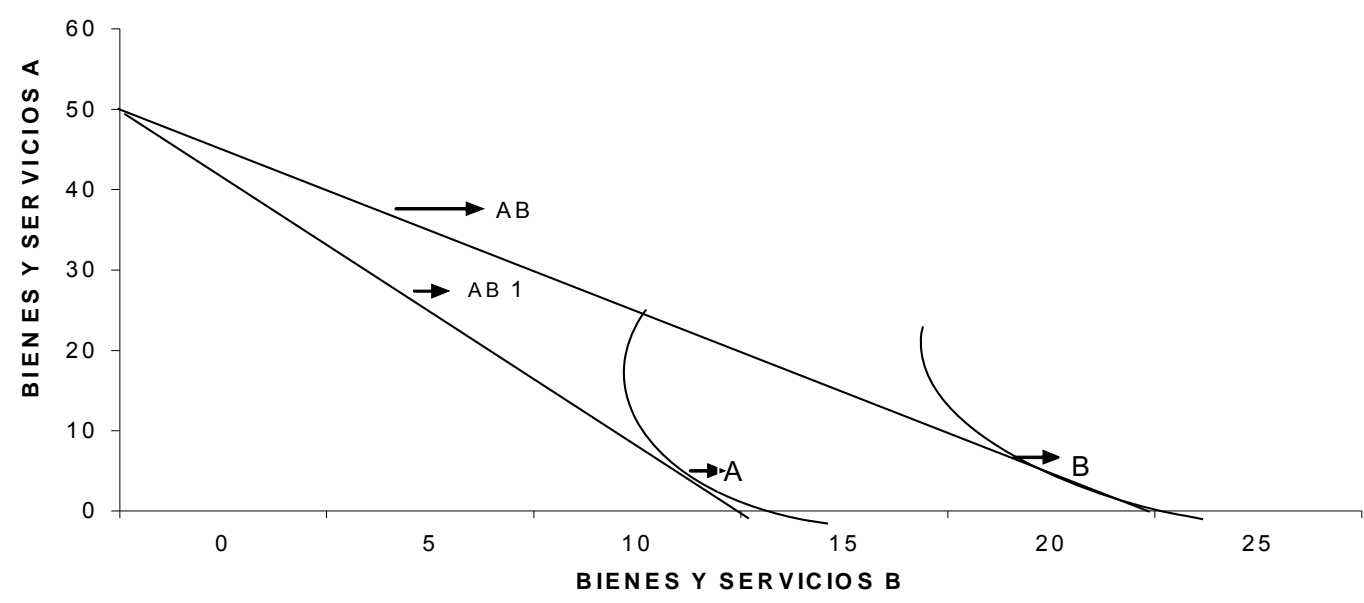

Fuente y elaboración propia

En la Recta de Restricción Presupuestaria AB, el consumidor se encontraba en la situación primigenia, es decir, que los precios de los productos no habían sufrido alteración alguna ni tampoco como se puede inferir el valor de su renta. La curva de indiferencia B es tangente con la Recta de Balance en el punto cuyas coordenadas son $(20,10)$ y es precisamente en dicho punto en donde el consumidor encontraba su equilibrio. Ahora bien, si se partiese de la premisa de que los precios de los bien(es) B se han duplicado, se observa que su nueva Recta de Balance AB sufre un desplazamiento en el eje de las abscisas hacia el punto de origen. Sin embargo, en el punto de las ordenadas permanece igual. Lo que explica que el consumidor seguirá teniendo las misma 
capacidad para adquirir el o los bien(es) A. El desplazamiento de la Recta de Balance hacia el punto de origen también explica que el consumidor se encuentra con una menor capacidad de adquisición de bien(es) B. Por otro lado, el consumidor encontrará su nuevo punto de equilibrio en las coordenadas $(10,10)$ punto en que la nueva curva de indiferencia A es tangente también a la nueva línea de Restricción presupuestaria AB 1. Es evidente, que en la curva de indiferencia A, los niveles de satisfacción del consumidor son inferiores a los de la curva de indiferencia B.

\section{Conclusiones}

1. El consumidor turístico, no escapa de las reglas generales del mercado, ni menos a su comportamiento microeconómico. Desde esta perspectiva, la recta de restricción presupuestaria del mismo, se puede ver ampliada si se considera el aspecto crediticio. Siempre y cuando dentro del análisis microeconómico se considere al dinero como una mercancía.

2. Debemos considerar que un individuo, cuando se propone realizar una actividad turística, por lo general evalúa mas de una alternativa o destino. Por lo general, el conjunto de destinos evaluados le significan similares expectativas y por lo tanto espera para cada uno de los destinos iguales niveles de satisfacción, los mismos que quedan plasmados en una curva de indiferencia.

3. Con respecto a lo señalado en la conclusión número dos, debemos también señalar el equilibrio del presupuesto del consumidor, combinado con el máximo nivel de satisfacción se encontrará en el punto de tangencia entre su recta de presupuesto y su curva de indiferencia. Dicho punto será uno y solo uno.

4. Cuando un consumidor afronta una disminución de sus ingresos, es obvio que su presupuesto de compras se vea restringido.

5. En lo referente al efecto precio, si un conjunto de bienes y servicios se ve incrementado con respecto a otros, es de esperarse que la capacidad de consumo de aquel conjunto se vea disminuida.

\section{Bibliografía recomendada}

B.C.R.P. Recuperado de www.bcrp.gob.pe

Barros de Castro, A., \& Lessa, C. (1995). Introducción a la economía. Un enfoque estructuralista. México: Siglo Veintiuno Editores.

Marsano, J. M. E. (2003). Economía del Turismo. Lima: Universidad de San Martín de Porres.

Monitor (1995). Turismo en el Perú. Lima: Comisión de Promoción del Perú.

Samuelson, P., \& Nordhaus, W. (1996). Economía. Madrid: McGraw-Hill. 